\title{
Crystal structures of two Bacillus carboxylesterases with different enantioselectivities
}

\author{
Henriëtte J. Rozeboom a , Luis F. Godinho ${ }^{\mathrm{b}, 1}$, Marco Nardini ${ }^{\mathrm{a}, 2}$, Wim J. Quax ${ }^{\mathrm{b}}$, Bauke W. Dijkstra ${ }^{\mathrm{a}, *}$ \\ a Laboratory of Biophysical Chemistry, Centre of Life Sciences, University of Groningen, Nijenborgh 7, 9747 AG Groningen, The Netherlands \\ b Department of Pharmaceutical Biology, Groningen Research Institute of Pharmacy, University of Groningen, Antonius Deusinglaan 1, 9713AV Groningen, The Netherlands
}

\section{A R T I C L E I N F O}

Article history:

Received 4 November 2013

Received in revised form 2 January 2014

Accepted 5 January 2014

Available online 11 January 2014

\section{Keywords:}

$\alpha / \beta$ Hydrolase

Carboxylesterase

MCP hydrolase

Molecular modeling

X-ray crystallography

\begin{abstract}
A B S T R A C T
Naproxen esterase (NP) from Bacillus subtilis Thai I-8 is a carboxylesterase that catalyzes the enantioselective hydrolysis of naproxenmethylester to produce $S$-naproxen ( $\mathrm{E}>200)$. It is a homolog of CesA (98\% sequence identity) and CesB (64\% identity), both produced by B. subtilis strain 168 . CesB can be used for the enantioselective hydrolysis of 1,2-O-isopropylideneglycerol (solketal) esters ( $\mathrm{E}>200$ for IPG-caprylate). Crystal structures of $\mathrm{NP}$ and CesB, determined to a resolution of $1.75 \AA$ and $2.04 \AA$, respectively, showed that both proteins have a canonical $\alpha / \beta$ hydrolase fold with an extra $\mathrm{N}$-terminal helix stabilizing the cap subdomain. The active site in both enzymes is located in a deep hydrophobic groove and includes the catalytic triad residues Ser130, His274, and Glu245. A product analog, presumably 2-(2-hydroxyethoxy)acetic acid, was bound in the NP active site. The enzymes have different enantioselectivities, which previously were shown to result from only a few amino acid substitutions in the cap domain. Modeling of a substrate in the active site of NP allowed explaining the different enantioselectivities. In addition, Ala156 may be a determinant of enantioselectivity as well, since its side chain appears to interfere with the binding of certain $R$-enantiomers in the active site of NP. However, the exchange route for substrate and product between the active site and the solvent is not obvious from the structures. Flexibility of the cap domain might facilitate such exchange. Interestingly, both carboxylesterases show higher structural similarity to meta-cleavage compound (MCP) hydrolases than to other $\alpha / \beta$ hydrolase fold esterases.
\end{abstract}

(c) 2014 Elsevier B.V. All rights reserved.

\section{Introduction}

Nature has evolved a diverse range of esterases with high regio- and stereoselectivity to convert a broad variety of chemical compounds in various metabolic processes [1]. Because of their specificity and selectivity, they are frequently used in biotechnological applications, such as in the food industry, for the production of perfumes, and for the synthesis of pharmaceuticals [2].

Carboxylesterases (carboxylic acid ester hydrolases, E.C. 3.1.1.1.) are a subset of esterases that hydrolyze small, water-soluble ester containing molecules resulting in the formation of an alcohol and a carboxylic acid. The microorganism $B$. subtilis strain Thai I-8 produces an enantiospecific carboxylesterase, called naproxen esterase or carboxylesterase NP (NP), which can be applied as a biocatalyst for the enantioselective resolution of racemic 2-arylpropionates, such as the important non-steroidal

\footnotetext{
Abbreviations: ee, enantiomeric excess; CesA, carboxylesterase CesA; CesB, carboxylesterase CesB; MCP, meta-cleavage compound; IPG, 1,2-O-isopropylideneglycerol; $\mathrm{NP}$, carboxylesterase NP

* Corresponding author.

E-mail address: B.W.Dijkstra@rug.nl (B.W. Dijkstra).

1 Present address: Department of Biology, Center of Cell Biology, University of Aveiro, Campus de Santiago, 3810-193 Aveiro, Portugal.

2 Present address: Department of Biosciences, Via Celoria 26,University of Milano, 20133 Milano, Italy.
}

anti-inflammatory drugs naproxen, ibuprofen, indomethacin, and nabumetone. NP has a product ee of $96 \%$ based on the substrate $S$-naproxenmethylester (Fig. 1) and 99\% on S-naproxenethylester (E > 200) [3,4]. In contrast, the enzyme's enantioselectivity towards 1,2-O-isopropylideneglycerol esters (IPG esters) (Fig. 1) is not sufficient for industrial processes (E values of $~ 1.2$ for $\mathrm{C} 4$ and 1.3 for C8 IPG esters, respectively) [5]. (S)-IPG (D-(+)-solketal) is an important building block for the synthesis of the biologically active forms of several well-known pharmaceuticals and endogenous compounds such as $\beta$-blockers, prostaglandins, and leukotrienes $[5,6]$.

B. subtilis strain 168 contains two homologs of NP called CesA (98\% identical to NP; also known as carboxylesterase NA) and CesB (64\% identical to NP; also known as YbfK). Unlike NP, CesB does convert $(S)$-IPG-caprylate ester with high enantioselectivity to $(S)$-IPG (ee of $99.9 \%, \mathrm{E}>200$ ), but not (R)-IPG-caprylate ester (Fig. 1) [5]. No marked differences in activity and selectivity were observed between NP and CesA [7].

All carboxylesterases of which 3D-structures have been elucidated to date share high structural similarity; all contain the characteristic $\alpha / \beta$ hydrolase fold (see the ESTHER database) [8]. This fold is composed of a central $\beta$-sheet flanked on both sides by $\alpha$-helices and serves as a stable protein core where, during evolution, amino acid substitutions, loop insertions or deletions have led to enzymes with diverse catalytic functions [9-13]. 
<smiles>CCCCCCCC(=O)OC[C@H]1COC(C)(C)O1</smiles>

(S)-IPG-caprylate<smiles>COC(=O)C(C)c1ccc2cc(OC)ccc2c1</smiles>

(S)-Naproxen methyl ester<smiles>CC1(C)OC[C@@H](CO)O1</smiles>

(S)-IPG

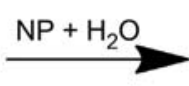<smiles>CCCCCCCC(=O)O</smiles>

Caprylate<smiles>COc1ccc2cc(C(C)C(=O)O)ccc2c1</smiles>

(S)-Naproxen

Fig. 1. Two-dimensional depiction of the reaction catalyzed by the carboxylesterases. The * indicates the chiral carbon atom.

Members of the $\alpha / \beta$ hydrolase fold have a nucleophile-His-acid catalytic triad with a sequence order of nucleophile, acid, and histidine [14]. In carboxylesterases the nucleophile is a serine residue, while the acid can be an aspartate or a glutamate residue. The serine residue is the central residue of the conserved pentapeptide sequence motif Gly-X1-Ser-X2-Gly [1], which defines a 'nucleophilic elbow' characterized by a very sharp turn between strand $\beta 5$ and the following $\alpha$-helix. In the discussed carboxylesterases this motif is Gly-Leu-Ser-Leu-Gly.

The catalytic mechanism proposed for carboxylesterases starts with nucleophilic attack by the serine hydroxyl group on the substrate carbonyl carbon atom of the scissile bond. The serine hydroxyl group is activated by the catalytic histidine/acid pair, which takes up the proton from the Ser $\mathrm{OH}$ group. A transient tetrahedral intermediate is formed, which is stabilized by two peptide nitrogen atoms, usually from residues on strands $\beta 5$ and $\beta 3$, forming the so-called "oxyanion hole". The proton is then transferred from the histidine to the leaving alcohol group, while the acid group of the substrate becomes covalently bound to the serine forming the covalent intermediate. Next, the histidine activates a water molecule, which hydrolyzes the covalent intermediate via nucleophilic attack on the carbonyl carbon of the intermediate. After the hydrolysis, the histidine donates its proton back to the serine, and the acyl component of the substrate is released $[11,15]$.

Although the catalytic machinery of $\alpha / \beta$ hydrolases is very similar, the means by which they bind substrates varies from protein to protein. In most cases, the "cap" or "lid" domain, which shields the catalytic triad, contributes to substrate binding [12]. This domain is usually inserted at the $C$-terminal ends of strands $\beta 4, \beta 6$, $\beta 7$, or $\beta 8$, and may differ considerably in size in the various enzymes [12].

Here, we report the crystal structure of NP at 1.75 Å resolution, with a product analog in the active site, as well as the crystal structure of CesB at $2.0 \AA$ resolution. Surprisingly, the NP and CesB structures resemble more those of the $C-C$ bond cleaving (MCP) hydrolases than the $\alpha / \beta$ hydrolase fold esterases. Modeling of a substrate in the active site of NP allowed to defining the residues that determine their different enantiopecificities, in agreement with previous site-directed mutagenesis results [6].

\section{Materials and methods}

2.1. Expression, purification, crystallization and structure determination of NP

The nap gene coding for NP from B. subtilis Thai I-8 (CBS 679.85) was cloned into B. subtilis I-85. Cells were grown in $2 \times$ YT medium and harvested by centrifugation. After treatment of the cells with lysozyme and DNAse, the soluble fraction was harvested by centrifugation. Proteins were precipitated with $60 \%$ ammonium sulfate and redissolved in $0.02 \mathrm{M}$ MOPS buffer, pH 7.5, and ultrafiltrated with an Amicon YM filter.
The resulting solution was applied to an analytical HPLC-SEC column (TSK 2000 SW, 2 times $300 \times 7.5 \mathrm{~mm}$ ), and the protein was eluted with $0.01 \mathrm{M}$ MES (2-(N-morpholino)ethane sulfonic acid), pH 5.6, and $0.1 \mathrm{M} \mathrm{NaCl}$ with a flow rate of $1 \mathrm{ml} / \mathrm{min}$. The purified enzyme was lyophilized [16].

Crystallization of naproxen esterase was performed as described earlier [17]. In short, hexagonal plate-like crystals were grown by liquid-liquid diffusion by filling glass capillaries with $5-10 \mu \mathrm{l}$ of $80 \%$ $(\mathrm{w} / \mathrm{v})$ PEG 6000 in $0.1 \mathrm{M}$ Tris- $\mathrm{HCl}, \mathrm{pH} 8.0$, and closing them on one side. The equivalent amount of the protein solution $(10 \mathrm{mg} / \mathrm{ml})$ in $0.1 \mathrm{M}$ Tris- $\mathrm{HCl}$ was added on top of the PEG column and then the capillaries were closed. The temperature was gradually increased over a four-week period from $4{ }^{\circ} \mathrm{C}$ to room temperature.

Crystals were mounted in a cryoloop and flash-cooled, without adding any cryoprotectant, prior to data collection. Data were collected on the BW7B beamline of the EMBL outstation at DESY (Hamburg, Germany) at $100 \mathrm{~K}$. Intensity data were processed using DENZO and SCALEPACK [18]. The crystal diffracted to $1.75 \AA$ And belonged to the trigonal space group $\mathrm{P}_{2} 21$ (Table 1 ), with one monomer of $33.8 \mathrm{kDa}$ in the asymmetric unit (corresponding to a calculated $V_{M}$ of $2.1 \AA 3 / \mathrm{Da}$ [19], and an estimated solvent content of 40\%).

The crystal structure of NP was solved by molecular replacement with PHASER [20], using a search model composed of three homologous proteins, which were suggested by the Fold and Function Assignment System (FFAS) server [21]. The search model was composed of the structures of Pseudomonas putida esterase (PDB ID: 1ZOI) [22], the metacleavage product hydrolase (CumD) from Pseudomonas fluorescens IP01 (PDB ID: 1IUN) [23], and the 2-hydroxy-6-oxo-6-phenylhexa-2,4dienoate hydrolase (BphD) from Rhodococcus sp. strain RHA1 (PDB ID: 1C4X) [24], superimposed on each other.

Several cycles of manual re-building with Coot [25], automatic model building with ARP/wARP [26], and refinement with REFMAC5 [27] were performed in order to improve and to expand the original model of 125 amino acid residues distributed in $6 \beta$-strands and $5 \alpha$ helices. Subsequently, a strategy to avoid model bias was applied, employing density modification, solvent flattening, statistical phase improvement, and automated model building performed in cycles using RESOLVE [28]. The best partial model generated by RESOLVE contained 180 amino acid residues with additional interpretable electron density, suitable for manual building. The model was completed by a few cycles with ARP/wARP and was further refined with REFMAC5 using TLS refinement [29]. Water molecules were automatically added by Coot, and manually checked. A 2-(2-hydroxyethoxy)acetic acid molecule was included at the last stage of refinement in the proximity of the nucleophile Ser130. The final model consists of 283 amino acid residues (9-293), 220 water molecules and one 2-(2-hydroxyethoxy)acetic acid molecule and has a $R$ factor of $22.4 \%$ and $R_{\text {free }}$ of $26.4 \%$ (Table 1 ). The first 8 and last 7 residues are not visible in the electron density and therefore not included in the final model. The stereochemical 
Table 1

Data collection and refinement statistics.

Values in parentheses are for the highest resolution shell.

\begin{tabular}{|c|c|c|}
\hline Data collection & NP & CesB (YbfK) \\
\hline Space group & $P 3_{2} 21$ & $P 2$ \\
\hline Cell dimensions $a, b, c(\AA)$ & $47.0,47.0,212.5$ & $82.1,44.0,108.7$ \\
\hline$\alpha, \beta, \gamma\left({ }^{\circ}\right)$ & $90.0,90.0,120.0$ & $90.0,91.9,90.0$ \\
\hline Resolution range $(\AA)$ & $40.0-1.75$ & $46.0-2.04$ \\
\hline Number of measurements & 134364 & 154210 \\
\hline Number of unique reflections & 28534 & 47148 \\
\hline$R_{\text {merge }}^{\dagger}(\%)$ & $5.7(20.4)$ & $13.3(39.4)$ \\
\hline Completeness (\%) & $99.4(100.0)$ & $92.8(76.1)$ \\
\hline Overall I/ $\sigma(\mathrm{I})$ & $21.4(5.4)$ & $7.1(2.9)$ \\
\hline \multicolumn{3}{|l|}{ Refinement } \\
\hline$R_{\text {cryst }} / R_{\text {free }}{ }^{g}(\%)$ & $22.7 / 26.7$ & $20.4 / 25.0$ \\
\hline \multicolumn{3}{|l|}{ Geometry } \\
\hline R.m.s. deviation bonds $(\AA)$ & 0.008 & 0.008 \\
\hline R.m.s. deviation angles $\left({ }^{\circ}\right)$ & 1.2 & 1.2 \\
\hline \multicolumn{3}{|l|}{ Contents of asymmetric unit } \\
\hline No. of protein atoms & 2290 & 4500 \\
\hline No. ligand atoms & 1 2-(2-hydroxyethoxy)acetic acid & 4 sodium ions \\
\hline No. water atoms & 220 & 461 \\
\hline Av. main chain B-factor & 33.9 & $17.6 / 20.0$ \\
\hline Av. ligand B-factor & 49.5 & - \\
\hline Av. Water B-factor & 40.3 & 26.1 \\
\hline \multicolumn{3}{|l|}{ Ramachandran plot } \\
\hline Favored regions (\%) & 96.8 & 96.5 \\
\hline Outliers (\%) & 0.4 & 0.0 \\
\hline \multicolumn{3}{|l|}{ Molprobity } \\
\hline Clashscore/percentile & $7.1 / 88^{\text {th }}$ & $5.2 / 97^{\text {th }}$ \\
\hline Molprobity score/percentile & $1.58 / 89^{\text {th }}$ & $1.51 / 97^{\text {th }}$ \\
\hline PDB ID & $4 \mathrm{CCW}$ & $4 C C Y$ \\
\hline
\end{tabular}

$R_{\text {merge }}={ }_{h k l i}\left|I_{i}(h k l)-\langle I(h k l)\rangle\right| \div{ }_{h k l} I_{i}(h k l)$.

${ }^{9} R_{\text {cryst }}={ }_{h k l}\left|F_{\text {obs }}-F_{\text {calc }}\right| \div{ }_{h k l} F_{\text {obs }}$, where $F_{\text {obs }}$ is the observed structure factor and $F_{\text {calc }}$ is the calculated structure factor. $R_{\text {free }}$ is the same as $R_{\text {cryst }}$, except that it was calculated using $5 \%$ of the data that were not included in any refinement calculations.

quality of the model was assessed with MolProbity [30]. Residue Ser175 has phi/psi angles in the disallowed region of the Ramachandran plot. This strained conformation is due to crystal contacts.

\subsubsection{Modeling of the enzyme-substrate complex}

Modeling of an enzyme-substrate complex was performed with the program Coot. The general position of the tetrahedral intermediate was modeled by superposing the catalytic triad of NP with the catalytic triad of carboxylesterase Est30 from Geobacillus stearothermophilus in complex with a covalently bound tetrahedral reaction intermediate (propyl actetate) (PDB ID: 1TQH) [31]. Est30 has 16\% overall sequence identity to NP (Table S1).

Atomic coordinates and structure factor amplitudes have been deposited with the RCSB Protein Data Bank with accession code 4CCW for carboxylesterase NP.

\subsection{Expression, purification, crystallization and structure determination of CesB}

The CesB gene was amplified from the pMAybfkvector [7] using two synthetic primers. The forward primer (5'-GGGAGGGGCATT CCATGG TACAAGATTC- $3^{\prime}$ ) contained an NcoI restriction site (in bold) and the reverse primer (5'-CTTTTCATG AAGCTT TTCCTTACTATTTTATC-3') contained a HindIII restriction site (in bold). The resulting PCR product and the $\mathrm{pBAD} / \mathrm{Myc}-\mathrm{HisA}$ vector were digested with NcoI and HindIII restriction enzymes, purified, and ligated using T4 DNA Ligase. The ligation mixture was used to transform competent Escherichia coli TOP10 cells. To verify the presence of the insert, transformants were selected from LB/Amp plates, and analyzed by colony PCR. Positive clones were grown overnight on LB/Amp medium and the plasmid DNA was isolated for sequence analysis.

The vector $\mathrm{pBAD} / \mathrm{Myc}$-His A was used for the expression of wild type CesB. E. coli TOP10 cells containing the appropriate expression plasmid were inoculated in $2 \times \mathrm{LB} / \mathrm{Amp}$ medium $(5 \mathrm{ml})$ and grown overnight at $37{ }^{\circ} \mathrm{C}$. Next day the $\mathrm{A}_{600}$ was determined for the overnight cultures and a sufficient quantity of the culture was used to inoculate $2 \times \mathrm{LB} /$ Amp medium ( $1 \mathrm{~L})$ to an initial $A_{600}$ of 0.05 . Cultures were grown at $37{ }^{\circ} \mathrm{C}$ till $\mathrm{A}_{600} \geq 0.5$, when the cultures were induced with arabinose $(0.02 \%, \mathrm{w} / \mathrm{v})$ and the temperature was changed to $20^{\circ} \mathrm{C}$. After $16 \mathrm{~h}$ cells were harvested and resuspended in $3 \mathrm{~mL} / \mathrm{g}$ of wet cells in extraction buffer (10 mM Tris-HCl, pH 8, 1 mM EDTA). Cells were disrupted by sonication, and extracts were clarified by centrifugation (50 min, $40,000 \mathrm{~g}$ ). Purification was performed by anion exchange chromatography using 3 HitrapQ columns $(1.6 \times 2.5 \mathrm{~cm})$ with $10 \mathrm{mM}$ Tris- $\mathrm{HCl}$, $\mathrm{pH}$ 8.0, and $1 \mathrm{mM}$ EDTA as Buffer A, and Buffer A containing $1.0 \mathrm{M}$ $\mathrm{NaCl}$ as Buffer B. Further purification was achieved by phenylsepharose HP chromatography with $10 \mathrm{~mm}$ phosphate buffer, $\mathrm{pH}$ 8.0, containing $0.5 \mathrm{M}$ ammonium sulfate as Buffer $\mathrm{A}$, and Buffer B containing $10 \mathrm{~mm}$ phosphate buffer $\mathrm{pH} 8.0$ [5]. In addition, gel filtration using a Hiload Superdex 200 column (GE Healthcare) was used to determine the oligomeric state of CesB in solution (monomer) and for buffer exchange (50 mM Tris $\mathrm{HCl}, 10 \%$ (v/v) glycerol, $\mathrm{pH} 8.0$ ).

CesB crystals were obtained from sitting-drop experiments with $20 \%$ polyethylene glycol 3350 and $0.2 \mathrm{M}$ sodium fluoride in $100 \mathrm{mM}$ BisTris propane buffer ( $\mathrm{pH}$ 6.0-7.0) as precipitant, using drops of $0.1 \mu \mathrm{l}$ of protein solution $\left(5.2 \mathrm{mg} \mathrm{ml}^{-1}\right.$ in $20 \mathrm{mM}$ Tris buffer, pH 7.5, $100 \mathrm{mM}$ $\mathrm{NaCl}$ ) and $0.1 \mu \mathrm{l}$ of reservoir solution set up by the Mosquito crystallization robot. Crystals grew in space group P2 (Table 1) with two monomers of $35.0 \mathrm{kDa}$ in the asymmetric unit (corresponding to a calculated $V_{\mathrm{M}}$ of $2.8 \AA^{3} / \mathrm{Da}[19]$, and an estimated solvent content of $56 \%$ ).

Before data collection, crystals were soaked for $15 \mathrm{~s}$ in a cryoprotectant solution, consisting of reservoir solution supplemented with $15 \%$ glycerol, directly followed by flash cooling. X-ray data were collected in house at $110 \mathrm{~K}$ with a MarDTB Goniostat System using $\mathrm{Cu}-\mathrm{K} \alpha$ radiation from a Bruker MicrostarH rotating-anode generator equipped with HeliosMX mirrors. Intensity data were processed using iMosflm [32] and scaled using SCALA from the CCP4 suite [27].

The structure of CesB was solved by molecular replacement with PHASER [20] using the NP structure as a search model. The phases were improved with the ARP/WARP [26] procedure combined with manual model building with Coot [25]. TLS refinement [29] was performed with REFMAC5 [27] and resulted in a final model comprising 2 protein molecules of 285 residues each in the asymmetric unit, 2 sodium ions and 460 water molecules, with a final $R$ factor of $20.4 \%$ and $R_{\text {free }}$ of $25.0 \%$ (Table 1 ). No electron density was visible for the first 10 residues and the $\mathrm{C}$-terminal residues of both molecules and these residues were therefore not included in the final model. The stereochemical quality of the model was assessed with MolProbity [30]. The sodium ions were assigned by the STAN server [33].

Atomic coordinates and structure factor amplitudes of carboxylesterase CesB have been deposited with the RCSB Protein Data Bank with accession code 4CCY.

\section{Results and discussion}

\subsection{Overall structure}

The structures of $B$. subtilis Thai I-8 carboxylesterase NP and B. subtilis 168 CesB were solved by molecular replacement and refined to $1.75 \AA$ A resolution (space group $P 3_{2} 21$ ) and $2.04 \AA$ A resolution (space group $P 2$ ), respectively. Data collection and refinement statistics are summarized in Table 1. The asymmetric unit of the NP crystal contains one protein molecule of 283 amino acid residues whereas the asymmetric unit of the CesB crystal contains two protein molecules of 285 residues each. Both carboxylesterases have a globular shape with 
dimensions of $\sim 54 \times 51 \times 43 \AA^{3}$ and share a superimposable tertiary structure with a root mean square deviation (RMSD) value of $0.94 \AA$ for $273 \mathrm{C} \alpha$ pairs, as expected from their high sequence identity (64\%). Like most of the members of the $\alpha / \beta$ hydrolase fold family, the NP and CesB structures consist of two domains: a central $\alpha / \beta$ "core" domain, containing the catalytic triad, and an $\alpha$-helical "cap" region on top of it, responsible for substrate recognition/binding (Fig. 2). It should be noted that NP is $98.0 \%$ identical in sequence to $B$. subtilis strain 168 CesA, differing only in 6 amino acids (L101V, K181T, T188N, Q202K, $\mathrm{K} 217 \mathrm{~N}, \mathrm{~A} 282 \mathrm{~T}$ ) located on the surface of the protein and not influencing the specific activity of the proteins [7]. Recently a putative hydrolase (2632844) from B. subtilis strain 168 was deposited with the Protein Data Bank (PDB ID: 2R11) (http://dx.doi.org/10.2210/pdb2r11/pdb) as part of a structural genomics project (Joint Center for Structural Genomics). This enzyme has $100 \%$ sequence identity to B. subtilis CesA. As expected, the crystal structures of NP and CesA are very similar, having a RMSD value of $0.55 \AA$ Aver $282 \mathrm{C} \alpha$ pairs. CesB is also highly similar to these two enzymes, and therefore the structural description will focus on NP. Specific differences among the structures will be eventually highlighted.

The NP core domain comprises residues 30-157 and 224-300, and it consists of a central eight-stranded, predominantly parallel $\beta$-sheet flanked by one 310 -helix ( $\eta 2)$ and three $\alpha$-helices on one side ( $\alpha 2$, $\alpha 10$ and $\alpha 11)$ and by four $\alpha$-helices $(\alpha 3, \alpha 4, \alpha 8$ and $\alpha 9)$ and one $3_{10}$-helix ( $\eta 3$ ) on the other side (Figs. $2 \mathrm{a}, \mathrm{S} 1$ and S2). The core domain has two 310 -helices ( $\eta 2$ and $\eta 3$ ), a small $\beta$-strand $\left(\beta 4^{\prime}\right)$ and one small $\alpha$-helix $(\alpha 10)$ extra compared to the 'canonical' $\alpha / \beta$ hydrolase fold $[9,10,12]$. Additionally, in CesB a small antiparallel $\beta$-sheet is formed with $\beta 6^{\prime}$ and $\beta 6^{\prime \prime}$ (Figs. 2 b, S2). Furthermore, CesA misses $\eta 1$ and $\eta 4$ but has a small $\beta$-strand $\beta 6^{\prime}$.

The cap domain comprises the $\mathrm{N}$-terminal residues $1-29$ and residues 158-223 (inserted between strand $\beta 6$ and helix $\alpha 8$ ) and consists of four $\alpha$-helices ( $\alpha 1, \alpha 5, \alpha 6$ and $\alpha 7$ ) and one $3_{10}$-helix ( $\eta 4$ ) (Figs. 2a, $\mathrm{S} 2$ ). The $\eta 433_{10}$-helix is located before helix $\alpha 7$ in NP, while it is after $\alpha 7$ in CesB (Fig. 2B). In this class of esterases the most striking feature of the cap domain is the presence of the N-terminal helix $\alpha 1$, which is situated almost parallel to helix $\alpha 7$ and ends as a $3_{10}$-helix ( $\eta 1$ ). Interactions between $\alpha 1$ and $\alpha 7$ are mainly hydrophobic. An N-terminal $\alpha$-helical extension is rare for $\alpha / \beta$ hydrolase fold proteins, being observed only in the hormone-sensitive lipase subfamily of enzymes such as brefeldin A esterase from B. subtilis (PDB ID: 1JKM) [34], Est2 from Alicyclobacillus acidocaldarius (PDB ID: 1EVQ) [35,36], AFEST, a novel hyper-thermophilic carboxylesterase from the archaeon Archaeoglobus fulgidus (PDB ID: 1JJI) [37] and acetyl esterase HerE from Rhodococcus sp. strain H1 (PDB ID: 1LZL) [38]. In these four enzymes the cap domain is built up of two N-terminal $\alpha$-helices and two $\alpha$-helices from the insert located after strand $\beta 6$. Kinetic data of Est 2 indicate that the first 35 residues of the protein play a role in the conformational stability of the protein and have a regulatory role [36,39]. It is likely that the N-terminal $\alpha$-helix in the carboxylesterases has a similar function. A DALI search [40] with the $\alpha / \beta$ hydrolase core domain did come up with many structural neighbors (Table S1). In contrast, for the isolated cap domain no neighbors were found, which makes these carboxylesterases unique in the family of esterases.

The cap domain is also the protein region where most of the sequence variations are present between NP and CesB (Fig. S1). The most disparate part is at amino acids 209-218 where differences in amino acid sequence have direct structural implications, such as the presence of the 310 -helix $\eta 4$ (residues 210-212) in CesB but not in NP, or the narrower active site entrance in CesB (Fig. 3). Variations in sequence are also observed in the active sites of NP and CesB [6], explaining the different substrate specificities (see below).

\subsection{Sodium ion binding to CesB}

Two sodium ions, originating from the crystallization solution, are bound to each of the two CesB monomers present in the asymmetric unit (Fig. 4). The first sodium ion (Na1) (Fig. 4A) is bound in a pocket lined by residues 244 to 250 (loop between strand $\beta 7$ and helix $\alpha 9$ ). It is octahedrally coordinated by $0 \varepsilon 1$ of $\operatorname{Gln} 250$ ( $2.34 \AA$ ), the carbonyl oxygen atom of Glu245 (2.31 $\AA$ ), the carbonyl oxygen atom of Tyr248 ( $2.69 \AA$ ) , and three water molecules ( $2.59 \AA, 2.53 \AA$, and $2.46 \AA$ ). Interestingly, Glu245 is the acid member of the catalytic triad. There is no sodium ion bound in NP or CesA at this position, where Gln250 is substituted for a Pro. The other sodium ion (Na2) (Fig. 4B) is located $15 \AA$ Away from Na1; it also has an octahedral geometry, being coordinated by Thr1870 1 ( $2.48 \AA$ ), the carbonyl oxygen atom of Gly188 (2.32 $\AA$ ), the carbonyl oxygen atom of Tyr191 (2.45 $\AA$ ), and three water molecules ( $2.33 \AA$, $2.44 \AA$, and $2.33 \AA$ ). The coordinating residues are part of the loop connecting helices $\alpha 6$ and $\alpha 7$ of the cap domain. At this location no sodium ion is bound in NP or CesA; the structures in this region are the most divergent. The binding of sodium could be a result of the purification and crystallization procedures.
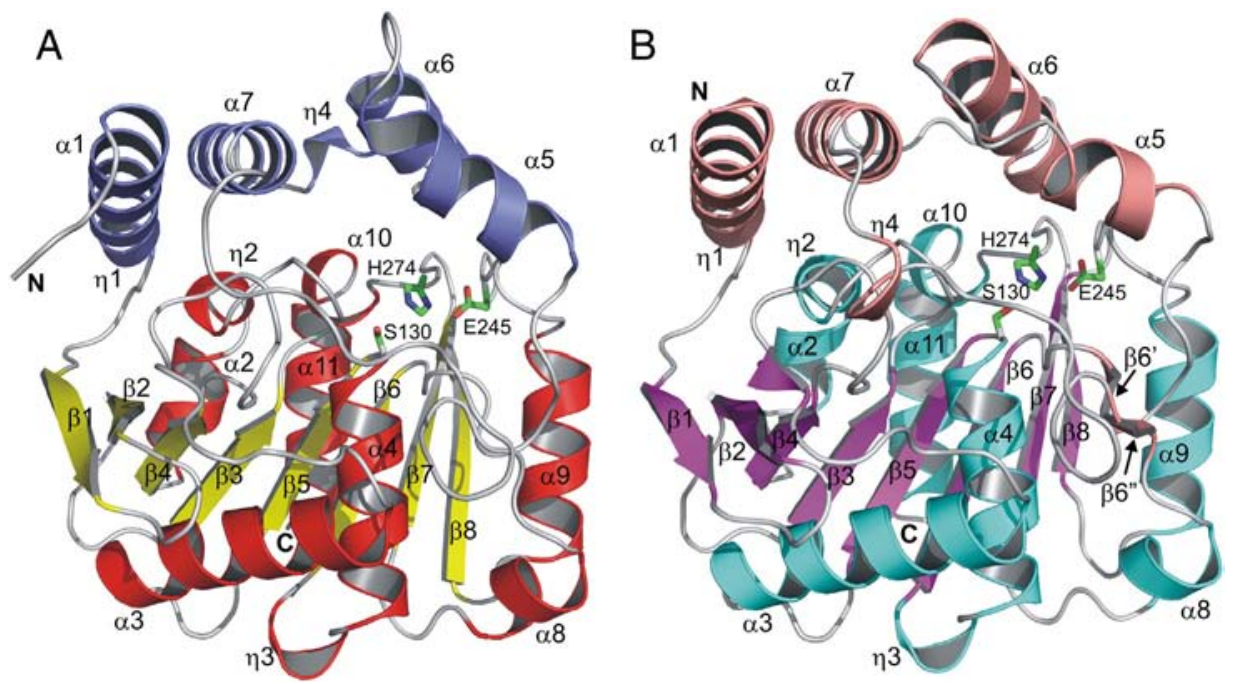

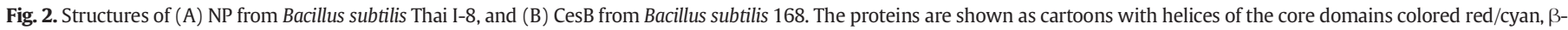

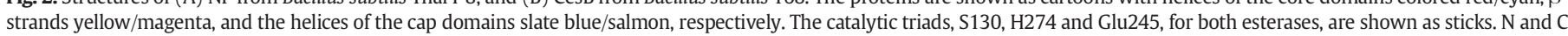
mark the amino and carboxylate termini of the proteins. 

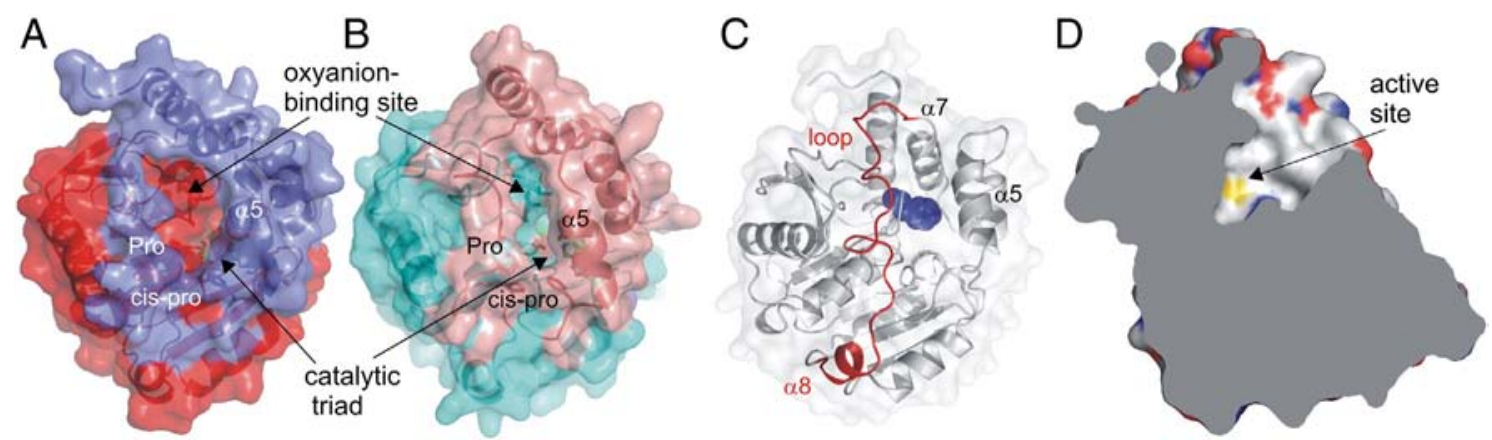

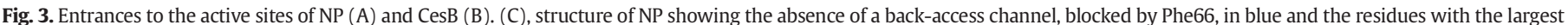
conformational differences between the carboxylesterases in red. (D), surface plot of NP showing one possible route to and from the active site.

\subsection{The active site}

The active site of NP is located at the interface between the core and the cap domains in a $\sim 13 \AA$ deep hydrophobic gorge. Its entrance is lined by residues from helices $\alpha 5, \alpha 6$, and $\alpha 7$ and from a loop located after helix $\alpha 7$, all belonging to the cap domain. The active site nucleophile Ser130 is located at the bottom of the gorge, protected from the bulk solvent, on the nucleophilic elbow between strand $\beta 5$ and helix $\alpha 4$ as part of the conserved sequence motif Gly-Leu-Ser-Leu-Gly. The main chain conformation of this serine is somewhat strained $\left(\varphi=53^{\circ}, \psi=-125^{\circ}\right)$, as more often observed in $\alpha / \beta$ hydrolases, but still in the allowed region of the Ramachandran plot. Its conformation is stabilized by a network of hydrogen bonds of the Ser130 carbonyl oxygen atom to the backbone amides of Gly132 (3.1 Å), Gly133 (2.9 Å), and Ala156 (3.0 ̊). The Ser130 backbone amide is at hydrogen bonding distance to the carbonyl oxygen atoms of Leu153 (3.0 $\AA$ ) and Ser154 (3.1 Å). Furthermore, in NP the Ser130 O y makes a hydrogen bond to the backbone amide of Leu131, which induces an unfavorable conformation of the nucleophile for the reaction to occur (Fig. 5A). On the contrary, in both CesB monomers the Ser130 O $\gamma$ is hydrogen bonded to the $\mathrm{N} \varepsilon 2$ atom of the catalytic His274 (2.8 $\AA$ ) (Fig. 5B). This observation suggests the presence of two populations existing for the Ser130 $0 \gamma$, one making a hydrogen bond to the backbone amide of Leu131 (representing the so called "resting" state, with a $\chi_{1}$ of $153^{\circ}$ ), another making a hydrogen bond to the His 274 side chain (the "active" state, with a $\chi_{1}$ of $-159^{\circ}$ ). Indeed, the catalytic His274 in $\alpha / \beta$ hydrolases is supposed to act as a base, deprotonating the serine to generate a very nucleophilic alkoxide $\left(-\mathrm{O}^{-}\right)$group. Interestingly, in CesA, where four independent molecules are present in the asymmetric unit, both populations are present, each associated with two monomers. The resting state found in NP is also reminiscent of that observed for the active site cysteine of dienelactone hydrolase [12]. Another example is given by $B$. subtilis lipase, although the resting state of the catalytic serine has a different conformation with a $\chi_{1}$ of $52^{\circ}$ [41]. These different conformations of the catalytic serine side chain support the notion that different orientations of its hydroxyl group are a necessary requirement for the enzymatic activity [41].

Regarding the third member of the catalytic triad, Glu245 is hydrogen bonded to His274, orienting its imidazole ring such that it interacts with Ser130. Having a glutamate residue as the third member of the catalytic triad is quite atypical; in other $\alpha / \beta$ hydrolases and serine esterases normally an aspartate residue is observed. A glutamate as member of the catalytic triad has been found in the family of large esterases (LES, with molecular weights above $55 \mathrm{kDa}$, subfamily VII [42]) like butyrylcholinesterases, acetylcholinesterases, human carboxylesterase and fungal lipases. Also Est713 [43], a smaller esterase of $35.5 \mathrm{kDa}$ has a glutamate as the third catalytic residue but it is positioned at the end of $\beta 6$, whereas in the carboxylesterases the glutamate is located at the end of $\beta 7$, the usual position for the catalytic acid residue in the $\alpha / \beta$ hydrolase fold enzymes.

Finally, in NP the amide NH groups of Ala64 (Gly64 in CesB) and Leu131 form the oxyanion-binding site that stabilizes the tetrahedral transition-state intermediates (Scheme 1).

\subsection{Product in the active site of carboxylesterase NP}

An unexpected residual electron density was observed in the active site of NP, near the side chain of Ser130 (Fig. 5A). This electron density is probably indicative of binding (part of) a chain of acidified PEG from the crystallization solution. The crystal was grown from 40\% PEG and was stored for several years in this solution before data were collected. It is known that polyethylene glycol solutions get oxidized by air after prolonged storage [44]. We modeled it as 2-(2-hydroxyethoxy)acetic acid. One of the carboxylate oxygen atoms is weakly hydrogen bonded

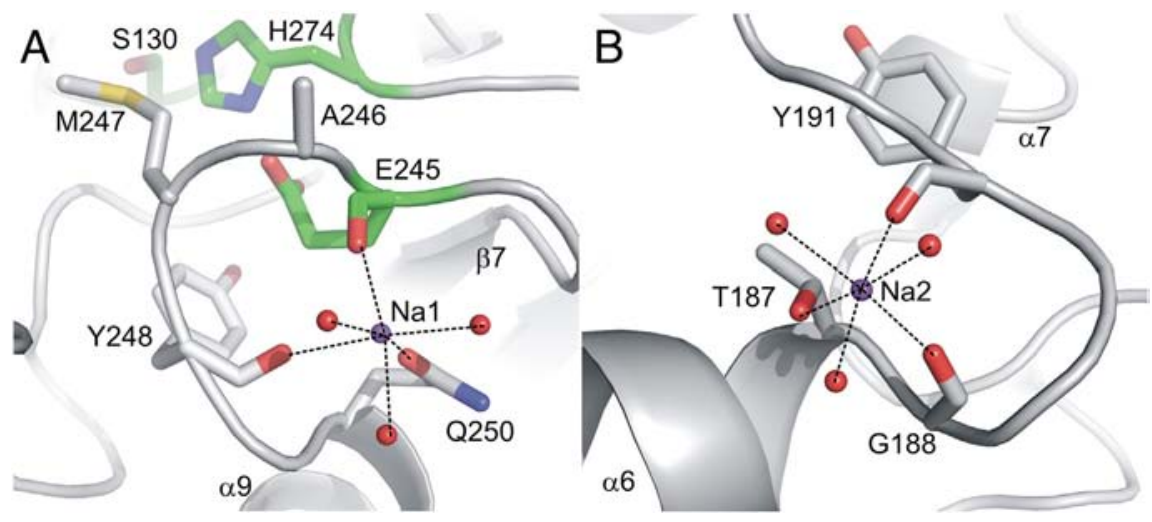

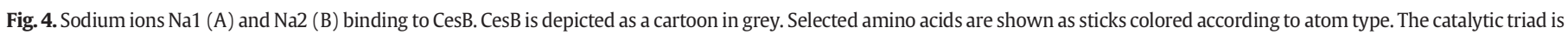
colored in green. Black dashed lines indicate interactions of the sodium ions with the amino acids and water molecules. 


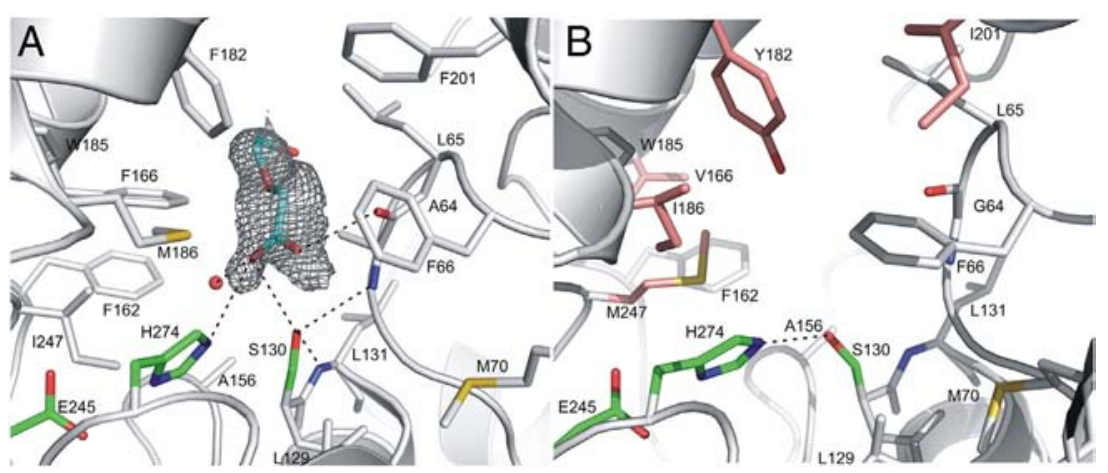

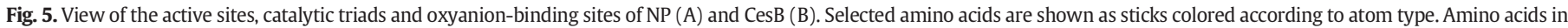

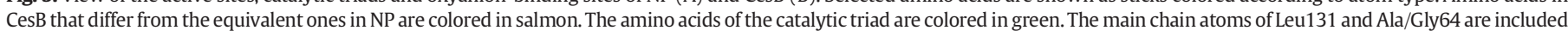
to mark the oxyanion-binding site. The product, 2-(2-hydroxyethoxy)acetic acid, is colored in cyan. The omit map is shown as a grey mesh and contoured at 10.

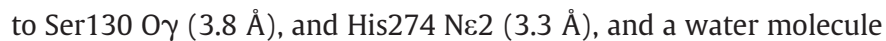
(3.4 $\AA$ ). The other carboxylate oxygen is hydrogen bonded to the carbonyl group of Ala64 (2.9 $\AA$ ), of which residue the amide nitrogen atom is involved in the oxyanion binding site (Fig. 5), but no interactions are present between the product analog and the oxyanion binding site amides. Several van der Waals interactions are present instead between the bound molecule and Ala64, Leu65, Phe166, Ala170 and Phe182 at the entrance of the active site.

In CesB only water molecules were found in the active site, but in CesA an ethyleneglycol molecule and a triethyleneglycol molecule were modeled in two of the four monomers in the asymmetric unit. The ethyleneglycol molecule in monomer B (active state) resides in about the same location as the product analog in NP; the triethyleneglycol molecule in monomer C (resting state) is also located at the entrance to the active site but bound via van der Waals interactions to Leu131 and Pro222. This shows the wide-open entrance to the active site of these carboxylesterases.

\subsection{Structural differences in the NP and CesB active sites and enantioselectivity properties}

While $(R, S)$-ethoxyethyl-[2-(6-methoxy-2-naphtyl)]propionate is enantioselectively hydrolyzed by NP to yield optically active $(S)$ naproxen, the enzyme hydrolyzes IPG-esters without enantioselectivity [7]. This lack of enantioselectivity towards IPG-substrates is mainly due to two residues, Phe166 and Phe182, in the active site as demonstrated

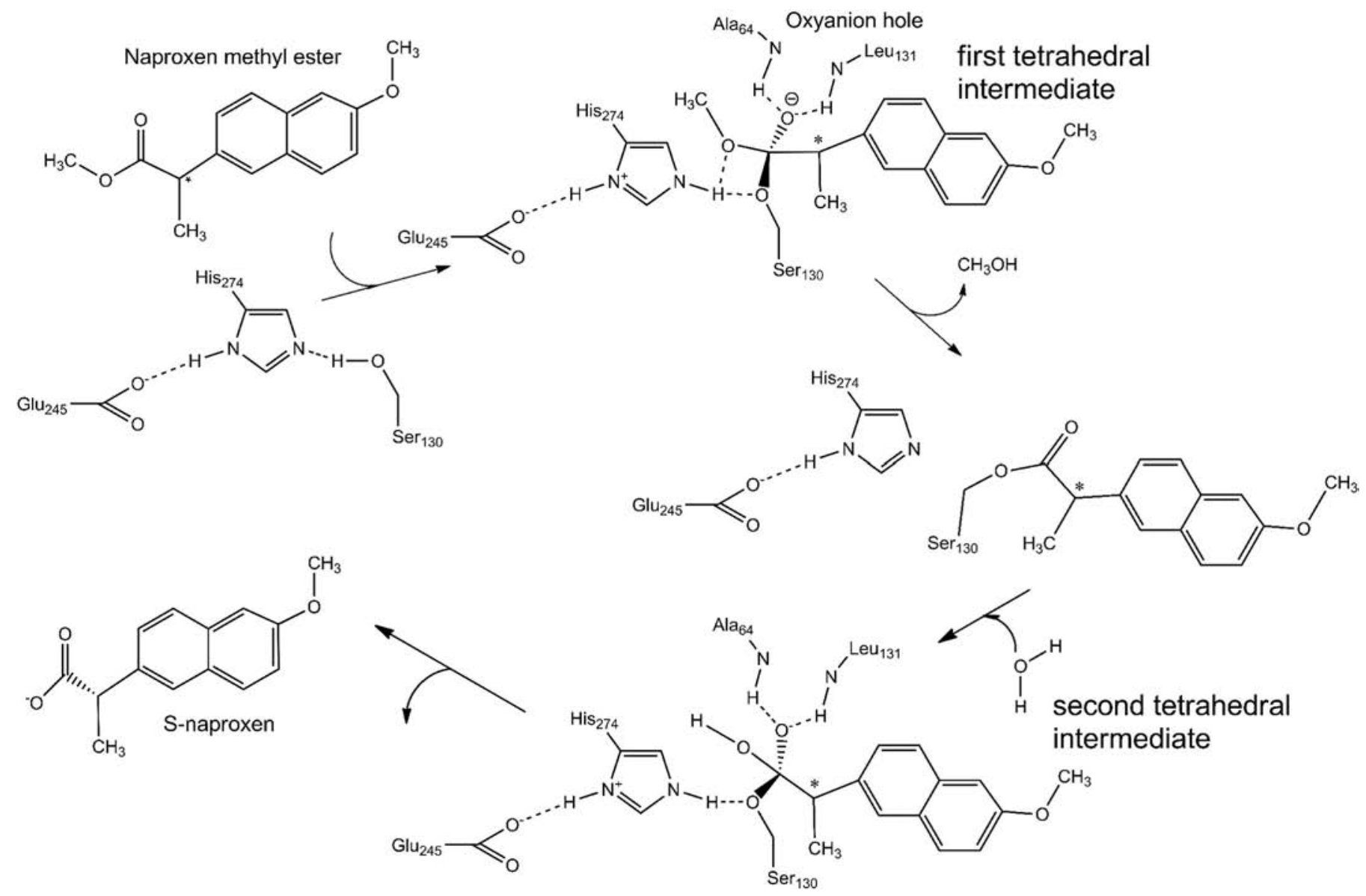

Scheme 1. Proposed mechanism of esterase-catalyzed hydrolysis of naproxen methyl ester. The chiral carbon in the substrate is marked with an * (asterisk). The figure has been adapted from Jaeger et al. [15]. 
by replacing them with the equivalent CesB residues Val166 and Tyr182 [6]. In contrast, CesB hydrolyzes 1,2-O-isopropylideneglycerol esters to (S)-IPG with 99.9\% enantioselectivity. Thus both esterases are highly selective, but NP is enantioselective towards the acyl/aryl part of substrates, while CesB specifically recognizes the chirality of the alcohol part, with some additional enantioselectivity towards the acyl/aryl part.

Since NP (and its homolog CesA) have a high enantiospecificity for $S$-naproxenethylester (99\%) [7], we tried to model the $S$ - and $R$ naproxenester substrates in the active site of NP (Fig. 6). For the modeling, several interactions were taken into account. For productive binding, the substrate's ester carbonyl oxygen atom needs to make hydrogen bonds to the two NH-groups of the oxyanion hole (Ala64 in NP, Gly64 in CesA, and Leu131 in both). Furthermore, the Nع2 atom of the catalytic His 274 should be at hydrogen bonding distance to the oxygen of the substrate alcohol and to the catalytic Ser130 O $\gamma$. In this configuration the substrate $S$-naproxenethylester binds with the alcohol moiety deep in the active site, and the naphtyl group at the hydrophobic entrance to the active site. Note, that the entrance to the active site is only $5.7 \AA$ wide, measured between $C \beta$ of Ala64 and $C \zeta$ of Phe166, and that it functions as a slit pocket. Using the same requirements, the model did not yield a productively bound $R$-naproxen ester substrate because of steric hindrance of the $R$-naproxen methyl group by Ala165, in agreement with the lack of activity of the enzyme towards this compound [7].

Applying the same criteria for productive binding, as used to model the naproxen ester in the active site of NP, the IPG moiety of the IPG esters most likely binds in the alcohol-binding pocket. The size of this pocket is mainly limited by Phe66 (Fig. 6), and could fit only methyl or ethyl substrates, in agreement with the enzyme's activity on naproxen methyl and ethyl esters [7]. However, the ability of the carboxylesterases to also hydrolyze the larger IPG esters [5,7] suggests that the active site alcohol-binding pocket is sufficiently flexible to accommodate the IPG-moiety. Only if Phe66, located at the interface between the two domains (Fig. 6A), adopts a different conformation, the alcohol-binding pocket would be able to accommodate the larger IPG alcohol moiety. Similar conformational changes have been observed in B. subtilis lipase $[45,46]$ where, upon binding of an IPG-like inhibitor, the alcohol-binding pocket expanded to accommodate the IPG moiety of the inhibitor.

CesB is enantioselective towards IPG caprylate ester [6], but NP is not. Indeed, our model shows that in CesB Tyr182 interacts with one of the IPG ring oxygen atoms, thus favoring the binding of the $(S)$ enantiomer over the $(R)$ enantiomer of IPG, where this hydrogen bond is not possible. In contrast, in NP the residue equivalent to Tyr182 is a Phe, which cannot make this hydrogen bonding interaction, resulting in a less selective binding.

It is noteworthy that in the carboxylesterases NP, CesB and CesA no separate entry and exit routes (backdoor, side-door, or tunnel) for substrate and product are observed. The exit route found in other esterases [47] is blocked by Phe162, His163, Pro164, Asp165 and Val166, all from the cap domain. Likewise, a possible water tunnel at the end of the alcohol-binding pocket is blocked by Phe66 (Fig. 3c,d). As a consequence, only one possible route to and from the active site is present in NP and CesB. However, in the reaction pathway the alcohol product is supposed to be the first to leave the active site (Scheme 1), but the exit route is blocked by the covalently bound acid part of the substrate. Similarly, the entrance route for the hydrolytic water molecule is obstructed. Yet, a lid-like movement of the cap domain (in particular of the flap made by helices $\alpha 5$ and $\alpha 6$ ) may be sufficient to allow the water molecule to enter the active site, and the products to be released. Indeed, the mainly hydrophobic interactions of the residues of helices $\alpha 5$ and $\alpha 6$ with the core domain could facilitate such a lid-like movement. Similar mechanisms of active site opening through cap domain helix relocation occur in family I.1 lipases $[48,49]$ as well as in other $\alpha / \beta$ hydrolases $[50,51]$.

3.6. Comparison to other $\alpha / \beta$ hydrolase fold esterases; similarity with meta-cleavage product hydrolases

A search of the Dali database [40] revealed that the NP and CesB structures are not very similar to those of other esterases, with the exception of the meta-cleavage product (MCP) hydrolases (Table S1). In general, the active site residues and oxyanion binding site amides are structurally conserved, but the alcohol-binding pockets of CesB and NP are smaller than those of other esterases with known structure.

In NP the acyl part of the substrate likely binds in the entrance to the active site as shown in Fig. 6 . In contrast, in other $\alpha / \beta$ hydrolase fold esterases the acyl part is usually bound in a deep hydrophobic pocket at the interface between the two domains $[31,51,52]$. However, at that position in NP, Ala156 and Glu157, two residues located at the domain interface, prevent binding of substrates containing a large acyl moiety. Instead, the acyl moiety binds in the entrance to the active site. This is possible, because the helices in the cap domain have a different position, causing the active site entrance of NP and CesB to have a different location compared to other esterases.

A secondary structure motif (SSM) search [53] of the PDB indicated significant structural similarity of the carboxylesterases NP and CesB with $\mathrm{C}-\mathrm{C}$ bond breaking enzymes that belong to the MCP hydrolase family [54]. These latter enzymes have 19-20\% sequence identity to NP (Table S1) and overall root mean square (r.m.s.) deviations of less than 2.3 Å. This superfamily of cytosolic hydrolases also includes soluble non-heme peroxidases, epoxide hydrolases, fluoroacetate dehalogenases and haloalkane dehalogenases [55]. Several MCP hydrolase family members also show esterase activity [56]. Intriguingly, compared to the dual specificity ester/MCP hydrolases, CesB and NP have an Ala156

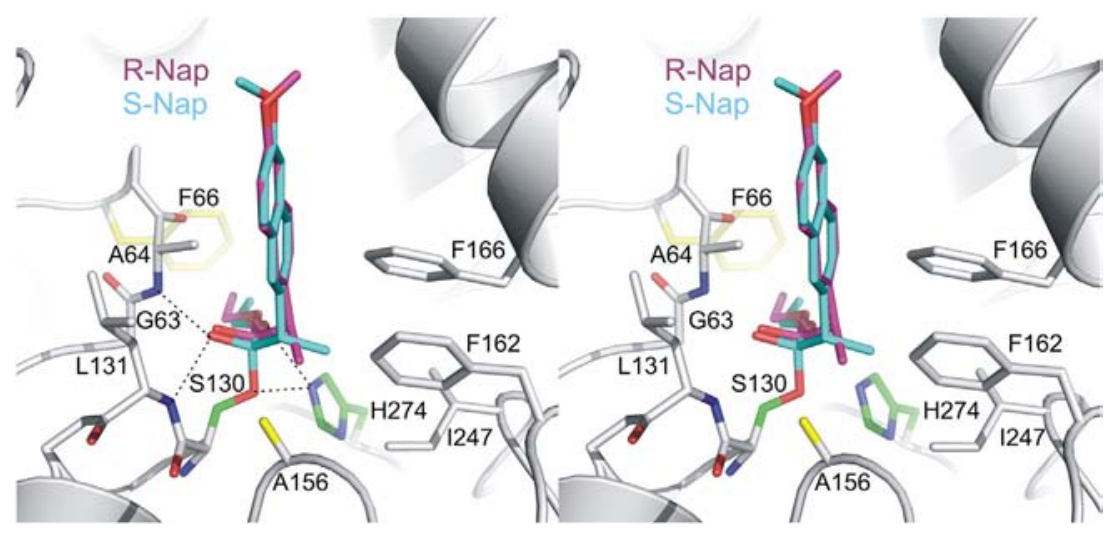

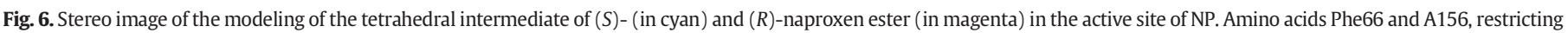
access to the active site, are colored in yellow. 
insertion, which extends the loop after $\beta 6$ and reduces the size of the active site, which probably contributes to their enantioselectivity.

Carboxylesterases and MCP hydrolases share a common position of the cap domain. In some MCP hydrolases the $\alpha$-helical lid (corresponding to residues 199-213 of CesB/NP) closes upon ligand binding [57] reducing the solvent accessibility of several hydrophobic residues. In NP and CesB residues 214-227, in the loop after helix $\alpha 7$, show the largest conformational differences compared to the MCP hydrolases (Fig. 3c). In fact, this is also the region where the structures of NP, CesA and CesB are most different. From these observations we conclude that the residues in this region may be flexible, which is also apparent from their elevated $B$-factor values. We suggest that this flexibility may be important to accommodate different substrates.

\section{Conclusions}

The structures of the B. subtilis carboxylesterases NP and CesB determined in the present study are very similar. Comparison of these structures to those of other $\alpha / \beta$ hydrolase fold esterases revealed that the main difference is the location of the entrance to active site, mainly because of a different positioning of the cap domain. In this respect NP and CesB resemble much more the MCP hydrolases, halogenases, dehalogenases and epoxide hydrolases than other esterases. Modeling of a substrate in the active site of NP indicates that the acyl chain is most likely bound at the entrance to the active site, unlike most other esterases, which have a well-defined pocket at the interface between the core and the cap domains.

Enantioselectivity of the carboxylesterases towards the IPGsubstrates is mainly due to a few residues in the active site, Phe166 and Phe182 in NP vs Val166 and Tyr182 in CesB. Ala156, an insertion in the carboxylesterases, may be a determinant of enantioselectivity as well, since its side chain appears to interfere with the binding of certain enantiomers in the active site of NP as shown by the substrate modeling.

In NP and CesB a lid domain movement for binding of the substrate and release of the product seems possible by movement of the flap made by helices $\alpha 5$ and $\alpha 6$ or/and of the loop after helix $\alpha 7$, where most of the amino acid and conformational differences between the three esterases occur. Such movements could also explain their activity on alcohol groups of the substrates that are too large to bind in the present conformation of the carboxylesterases.

Altogether, these observations make the carboxylesterases unique members of the esterase family, with their own specific enantiospecificities, and indicate an evolutionary relationship to the soluble meta-cleavage compound hydrolases.

Supplementary data to this article can be found online at http://dx. doi.org/10.1016/j.bbapap.2014.01.003.

\section{Acknowledgements}

We thank Johan Hekelaar for technical assistance with data collection of CesB crystals. We are grateful to the staff scientists at beam line BW7B at the EMBL-Hamburg outstation (DESY), Germany, for their support during data collection.

\section{References}

[1] U.T. Bornscheuer, Microbial carboxyl esterases: classification, properties and application in biocatalysis, FEMS Microbiol. Rev. 26 (2002) 73-81.

[2] K.E. Jaeger, T. Eggert, Lipases for biotechnology, Curr. Opin. Biotechnol. 13 (2002) 390-397.

[3] W.J. Quax, C.P. Broekhuizen, Development of a new Bacillus carboxyl esterase for use in the resolution of chiral drugs, Appl. Microbiol. Biotechnol. 41 (1994) 425-431.

[4] L. Steenkamp, D. Brady, Optimisation of stabilised Carboxylesterase NP for enantioselective hydrolysis of naproxen methyl ester, Process Biochem. 43 (2008) 1419-1426.

[5] M.J. Droge, R. Bos, W.J. Quax, Paralogous gene analysis reveals a highly enantioselective 1,2-0-isopropylideneglycerol caprylate esterase of Bacillus subtilis, Eur. J. Biochem. 268 (2001) 3332-3338.
[6] L.F. Godinho, C.R. Reis, H.J. Rozeboom, F.J. Dekker, B.W. Dijkstra, G.J. Poelarends, W.J Quax, Enhancement of the enantioselectivity of carboxylesterase A by structure-based mutagenesis, J. Biotechnol. 158 (2012) 36-43.

[7] M.J. Droge, R. Bos, Y.L. Boersma, W.J. Quax, Comparison and functional characterisation of three homologous intracellular carboxylesterases of Bacillus subtilis, J. Mol Catal. B Enzym. 32 (2005) 261-270.

[8] N. Lenfant, T. Hotelier, Y. Bourne, P. Marchot, A. Chatonnet, Proteins with an alpha/beta hydrolase fold: relationships between subfamilies in an ever-growing superfamily, Chem. Biol. Interact. 203 (2013) 266-268.

[9] D.L. Ollis, E. Cheah, M. Cygler, B. Dijkstra, F. Frolow, S.M. Franken, M. Harel, S.J Remington, I. Silman, J. Schrag, J.L. Sussman, K.H.G. Verschueren, A. Goldman, The alpha/beta-hydrolase fold, Protein Eng. 5 (1992) 197-211.

[10] M. Nardini, B.W. Dijkstra, alpha/beta hydrolase fold enzymes: the family keeps growing, Curr. Opin. Struct. Biol. 9 (1999) 732-737.

[11] M. Holmquist, Alpha/beta-hydrolase fold enzymes: structures, functions and mechanisms, Curr. Protein Pept. Sci. 1 (2000) 209-235.

[12] P.D. Carr, D.L. Ollis, Alpha/beta hydrolase fold: an update, Protein Pept. Lett. 16 (2009) 1137-1148.

[13] R. Kourist, H. Jochens, S. Bartsch, R. Kuipers, S.K. Padhi, M. Gall, D. Böttcher, H.J Joosten, U.T. Bornscheuer, The $\alpha / \beta$-hydrolase fold 3DM database (ABHDB) as a tool for protein engineering, Chembiochem 11 (2010) 1635-1643.

[14] P. Heikinheimo, A. Goldman, C. Jeffries, D.L. Ollis, Of barn owls and bankers: a lush variety of alpha/beta hydrolases, Structure 7 (1999) R141-R146.

[15] K.E. Jaeger, B.W. Dijkstra, M.T. Reetz, Bacterial biocatalysts: molecular biology, three-dimensional structures, and biotechnological applications of lipases, Annu. Rev. Microbiol. 53 (1999) 315-351.

[16] M.A. Bertola, W.J. Quax, B.W. Robertson, A.F. Marx, C.J. Van der Laken, H.S. Koger, G.T. Phillips, P.D. Watts, Microbial esterase and process for the preparation of 2-arylpropionic acids, 1987. (EP0233656).

[17] J.M. van der Laan, A.V. Teplyakov, A.A. Lammers, B.W. Dijkstra, Preliminary X-ray study of naproxen esterase from Bacillus subtilis, J. Mol. Biol. 230 (1993) 681-683.

[18] Z. Otwinowski, W. Minor, Processing of X-ray diffraction data collected in oscillation mode, Method Enzymol. 276 (1997) 307-326.

[19] B.W. Matthews, Solvent content of protein crystals, J. Mol. Biol. 33 (1968) 491-497.

[20] A.J. McCoy, R.W. Grosse-Kunstleve, P.D. Adams, M.D. Winn, L.C. Storoni, R.J. Read Phaser crystallographic software, J. Appl. Crystallogr. 40 (2007) 658-674.

[21] L. Jaroszewski, L. Rychlewski, Z.W. Li, W.Z. Li, A. Godzik, FFAS03: a server for profile-profile sequence alignments, Nucleic Acids Res. 33 (2005) W284-W288.

[22] F. Elmi, H.T. Lee, J.Y. Huang, Y.C. Hsieh, Y.L. Wang, Y.J. Chen, S.Y. Shaw, C.J. Chen, Stereoselective esterase from Pseudomonas putida IF012996 reveals alpha/beta hydrolase folds for D-beta-acetylthioisobutyric acid synthesis, J. Bacteriol. 187 (2005) 8470-8476.

[23] S. Fushinobu, S. Jun, M. Hidaka, H. Nojiri, H. Yamane, H. Shoun, T. Omori, T. Wakagi, A series of crystal structures of a meta-cleavage product hydrolase from Pseudomonas fluorescens IP01 (CumD) complexed with various cleavage products, Biosci. Biotechnol. Biochem. 69 (2005) 491-498.

[24] N. Nandhagopal, T. Senda, T. Hatta, A. Yamada, E. Masai, M. Fukuda, Y. Mitsui, Three-dimensional structure of microbial 2-hydroxyl-6-oxo-6-phenylhexa-2,4dienoic acid (Hpda) hydrolase (Bphd enzyme) from Rhodococcus Sp. strain RHA1, in the PCB degradation pathway, Proc. Jpn Acad. B 73 (1997) 154.

[25] P. Emsley, B. Lohkamp, W.G. Scott, K. Cowtan, Features and development of Coot Acta Crystallogr. D 66 (2010) 486-501.

[26] G. Langer, S.X. Cohen, V.S. Lamzin, A. Perrakis, Automated macromolecular mode building for X-ray crystallography using ARP/wARP version 7, Nat. Protoc. 3 (2008) 1171-1179.

[27] M.D. Winn, C.C. Ballard, K.D. Cowtan, E.J. Dodson, P. Emsley, P.R. Evans, R.M. Keegan, E.B. Krissinel, A.G. Leslie, A. McCoy, S.J. McNicholas, G.N. Murshudov, N.S. Pannu, E.A. Potterton, H.R. Powell, R.J. Read, A. Vagin, K.S. Wilson, Overview of the CCP4 suite and current developments, Acta Crystallogr. D 67 (2011) 235-242.

[28] T.C. Terwilliger, SOLVE and RESOLVE: automated structure solution and density modification, Methods Enzymol. 374 (2003) 22-37.

[29] J. Painter, E.A. Merritt, TLSMD web server for the generation of multi-group TLS models, J. Appl. Crystallogr. 39 (2006) 109-111.

[30] V.B. Chen, W.B. Arendall III, J.J. Headd, D.A. Keedy, R.M. Immormino, G.J. Kapral, L.W. Murray, J.S. Richardson, D.C. Richardson, MolProbity: all-atom structure validation for macromolecular crystallography, Acta Crystallogr. D 66 (2010) 12-21.

[31] P. Liu, Y.F. Wang, H.E. Ewis, A.T. Abdelal, C.D. Lu, R.W. Harrison, I.T. Weber, Covalent reaction intermediate revealed in crystal structure of the Geobacillus stearothermophilus carboxylesterase Est30, J. Mol. Biol. 342 (2004) 551-561.

[32] T.G. Battye, L. Kontogiannis, O. Johnson, H.R. Powell, A.G. Leslie, iMOSFLM: a new graphical interface for diffraction-image processing with MOSFLM, Acta Crystallogr. D 67 (2011) 271-281.

[33] M. Nayal, E. DiCera, Valence screening of water in protein crystals reveals potential $\mathrm{Na}^{+}$binding sites, J. Mol. Biol. 256 (1996) 228-234.

[34] Y.Y. Wei, J.A. Contreras, P. Sheffield, T. Osterlund, U. Derewenda, R.E. Kneusel, U. Matern, C. Holm, Z.S. Derewenda, Crystal structure of brefeldin A esterase, a bacterial homolog of the mammalian hormone-sensitive lipase, Nat. Struct. Biol. 6 (1999) 340-345.

[35] G. De Simone, S. Galdiero, G. Manco, D. Lang, M. Rossi, C. Pedone, A snapshot of a transition state analogue of a novel thermophilic esterase belonging to the subfamily of mammalian hormone-sensitive lipase, J. Mol. Biol. 303 (2000) 761-771.

[36] L. Mandrich, L. Merone, G. Manco, Structural and kinetic overview of the carboxylesterase EST2 from Alicyclobacillus acidocaldarius: a comparison with the other members of the HSL family, Protein Pept. Lett. 16 (2009) 1189-1200.

[37] G. De Simone, V. Menchise, G. Manco, L. Mandrich, N. Sorrentino, D. Lang, M. Rossi, C. Pedone, The crystal structure of a hyper-thermophilic carboxylesterase from the archaeon Archaeoglobus fulgidus, J. Mol. Biol. 314 (2001) 507-518. 
[38] X.Y. Zhu, N.A. Larsen, A. Basran, N.C. Bruce, I.A. Wilson, Observation of an arsenic adduct in an acetyl esterase crystal structure, J. Biol. Chem. 278 (2003) 2008-2014.

[39] L. Mandrich, L. Merone, M. Pezzullo, L. Cipolla, F. Nicotra, M. Rossi, G. Manco, Role of the $\mathrm{N}$ terminus in enzyme activity, stability and specificity in thermophilic esterases belonging to the HSL family, J. Mol. Biol. 345 (2005) 501-512.

[40] L. Holm, J. Park, DaliLite workbench for protein structure comparison, Bioinformatics 16 (2000) 566-567.

[41] K. Kawasaki, H. Kondo, M. Suzuki, S. Ohgiya, S. Tsuda, Alternate conformations observed in catalytic serine of Bacillus subtilis lipase determined at 1.3 A resolution, Acta Crystallogr. D 58 (2002) 1168-1174.

[42] J.L. Arpigny, K.E. Jaeger, Bacterial lipolytic enzymes: classification and properties, Biochem. J. 343 (1999) 177-183.

[43] P.C. Bourne, M.N. Isupov, J.A. Littlechild, The atomic-resolution structure of a novel bacterial esterase, Structure 8 (2000) 143-151.

[44] W.J. Ray, J.M. Puvathingal, A simple procedure for removing contaminating aldehydes and peroxides from aqueous-solutions of polyethylene glycols and of nonionic detergents that are based on the polyoxyethylene linkage, Anal. Biochem. 146 (1985) 307-312.

[45] G. van Pouderoyen, T. Eggert, K.E. Jaeger, B.W. Dijkstra, The crystal structure of Bacillus subtilis lipase: a minimal alpha/beta hydrolase fold enzyme, J. Mol. Biol. 309 (2001) 215-226.

[46] M.J. Droge, Y.L. Boersma, G. van Pouderoyen, T.E. Vrenken, C.J. Ruggeberg, M.T. Reetz, B.W. Dijkstra, W.J. Quax, Directed evolution of Bacillus subtilis lipase A by use of enantiomeric phosphonate inhibitors: crystal structures and phage display selection, Chembiochem 7 (2006) 149-157.

[47] J.D. Cheeseman, A. Tocilj, S. Park, J.D. Schrag, R.J. Kazlauskas, Structure of an aryl esterase from Pseudomonas fluorescens, Acta Crystallogr. D 60 (2004) 1237-1243.

[48] J.D. Schrag, Y. Li, M. Cygler, D. Lang, T. Burgdorf, H.J. Hecht, R. Schmid, D. Schomburg, T.J. Rydel, J.D. Oliver, L.C. Strickland, C.M. Dunaway, S.B. Larson, J. Day, A. McPherson, The open conformation of a Pseudomonas lipase, Structure 5 (1997) 187-202.
[49] M. Nardini, D.A. Lang, K. Liebeton, K.E. Jaeger, B.W. Dijkstra, Crystal structure of Pseudomonas aeruginosa lipase in the open conformation. The prototype for family I.1 of bacterial lipases, J. Biol. Chem. 275 (2000) 31219-31225.

[50] M. Otyepka, J. Damborsky, Functionally relevant motions of haloalkane dehalogenases occur in the specificity-modulating cap domains, Protein Sci. 11 (2002) 1206-1217.

[51] G. De Simone, V. Menchise, V. Alterio, L. Mandrich, M. Rossi, G. Manco, C. Pedone, The crystal structure of an EST2 mutant unveils structural insights on the $\mathrm{H}$ group of the carboxylesterase/lipase family, J. Mol. Biol. 343 (2004) 137-146.

[52] A. Jansson, J. Niemi, P. Mantsala, G. Schneider, Crystal structure of aclacinomycin methylesterase with bound product analogues: implications for anthracycline recognition and mechanism, J. Biol. Chem. 278 (2003) 39006-39013.

[53] E. Krissinel, K. Henrick, Secondary-structure matching (SSM), a new tool for fast protein structure alignment in three dimensions, Acta Crystallogr. D 60 (2004) 2256-2268.

[54] S.Y.K. Seah, J.Y. Ke, G. Denis, G.P. Horsman, P.D. Fortin, C.J. Whiting L.D. Eltis, Characterization of a C-C bond hydrolase from Sphingomonas wittichii RW1 with novel specificities towards polychlorinated biphenyl metabolites, J. Bacteriol. 189 (2007) 4038-4045.

[55] M. Fischer, J. Pleiss, The Lipase Engineering Database: a navigation and analysis tool for protein families, Nucleic Acids Res. 31 (2003) 319-321.

[56] M. Alcaide, J. Tornes, P.J. Stogios, X. Xu, C. Gertler, R. Di Leo, R. Bargiela, A. Lafraya, M.E. Guazzaroni, N. Lopez-Cortes, T.N. Chernikova, O.V. Golyshina, T.Y. Nechitaylo, I. Plumeier, D.H. Pieper, M.M. Yakimov, A. Savchenko, P.N. Golyshin, M. Ferrer, Single residues dictate the co-evolution of dual esterases: MCP hydrolases from the alpha/beta hydrolase family, Biochem. J. 454 (2013) 157-166.

[57] N.A. Lack, K.C. Yam, E.D. Lowe, G.P. Horsman, R.L. Owen, E. Sim, L.D. Eltis, Characterization of a carbon-carbon hydrolase from Mycobacterium tuberculosis involved in cholesterol metabolism, J. Biol. Chem. 285 (2010) 434-443.

[58] P. Gouet, E. Courcelle, D.I. Stuart, F. Metoz, ESPript: analysis of multiple sequence alignments in PostScript, Bioinformatics 15 (1999) 305-308. 